DRAFT VERSION JUNE 24, 2018

Preprint typeset using LATEX style emulateapj v. 11/12/01

\title{
THE ACCRETION RATIOS IN SEYFERT 2 GALAXIES WITH AND WITHOUT HIDDEN BROAD-LINE REGIONS
}

\author{
W. BIAN ${ }^{1,2}$ AND Q. GU ${ }^{3}$ \\ whbian@njnu.edu.cn \\ Received 2006 July 10; accepted 2006 Oct 30
}

\begin{abstract}
Using a large sample of 90 Seyfert 2 galaxies (Sy2s) with spectropolarimetric observations, we tested the suggestion that the presence of hidden broad-line regions (HBLRs) in Sy2s is dependent upon the Eddington ratio. The stellar velocity dispersion and the extinction-corrected [O III] luminosity are used to derive the mass of central super-massive black holes and the Eddington ratio. We found that: (1) below the Eddington ratio threshold of $10^{-1.37}$, all but one object belong to non-HBLRs Sy2s; while at higher Eddington ratio, there is no obvious discrimination in the Eddington ratio and black hole mass distributions for Sy2s with and without HBLRs; (2) nearly all low-luminosity Sy2s (e.g., $\left.L_{[\mathrm{O}} \mathrm{III}\right]<10^{41} \mathrm{erg} \mathrm{s}^{-1}$ ) do not show HBLRs regardless of the column density of neutral hydrogen $\left(N_{\mathrm{H}}\right)$; (3) for high-luminosity Sy2s, the possibility to detect HBLRs Sy2s is almost the same as that of non-HBLRs Sy2s; (4) when considering only Compton-thin Sy2s with higher [O III] luminosity $\left(>10^{41} \mathrm{erg} \mathrm{s}^{-1}\right)$, we find a very high detectability of HBLRs,$\sim 85 \%$. These results suggested that AGN luminosity plays a major role in not detecting HBLRs in low-luminosity Sy2s, while for high-luminosity Sy2s, the detectability of HBLRs depends not only upon the AGN activity, but also upon the torus obscuration.
\end{abstract}

Subject headings: galaxies: active — galaxies: Seyfert — galaxies: statistics

\section{INTRODUCTION}

Seyfert 2 galaxies (Sy2s) belong to a subclass of lowluminosity active galactic nuclei (AGN) with the absence of broad permitted optical lines, compared to Seyfert 1 galaxies (Sy1s). The standard paradigm of AGN is an accretion disk surrounding a central super-massive black hole (SMBH), with other components, such as broad-line regions (BLRs), narrowline regions (NLRs), jet, torus, et al. (e.g. Rees 1984; Antonucci 1993; Urry \& Padovani 1995). In the scheme of AGN oriented-unification model, the distinction between Sy2s and Sy1s depends on whether the central engine and BLRs are viewed directly (Sy1s) or are obscured by circumnuclear torus (Sy2s). This scenario was first suggested to explain the presence of polarized broad emission lines in NGC 1068, in addition to spectropolarimetric observations of hidden broad-line regions (HBLRs) in several other Sy2s (Antonucci \& Miller 1985; Tran 1995; Heisler, Lumsden \& Bailey 1997; Moran et al. 2000; Lumsden et al. 2001; Tran 2001; Zakamsa 2003b). Infrared observations also confirmed the existence of broad emission lines in several Sy2s (Veilleux, Goodrich \& Hill 1997). More evidence for this simple unification model comes from the X-ray observations: Sy2s show much higher column density of neutral hydrogen $\left(N_{\mathrm{H}}\right)$ than type 1 ones as expected from the torus obscuration. In the local Universe, about half of Sy2s are found to be Compton-thick sources with $N_{\mathrm{H}}>10^{24}$ $\mathrm{cm}^{-2}$ (Maiolino et al. 1998; Bassani et al. 1999; Risaliti, Maiolino \& Salvati 1999).

However, some Sy2s don't show HBLRs in the spectropolarimetric observations and some Sy2s have column densities lower than $10^{22} \mathrm{~cm}^{-2}$ (so called "unabsorbed Sy2s", Panessa \& Bassini 2002) in the X-ray observation, which challenged the oriented-unification model indeed. It is still not clear what kind of physical process is related to the presence of HBLRs in Sy2s. Natively, the detectability of HBLRs in Sy2s depends upon the sensitivity of observation, the strength of BLRs, and the inclination of the torus to the line of sight, etc (e.g. Moran et al. 2001; Gu \& Huang 2002; Nicastro et al. 2003; Laor 2003; Tran 2003). For unabsorbed Sy $2 \mathrm{~s}$ with $N_{\mathrm{H}}<10^{22} \mathrm{~cm}^{-2}$, their BLRs are either obscured by something other than the torus or it is weak or absent (Panessa \& Bassini 2002; Gallo et al. 2006). For Compton-thick Sy2s $\left(N_{\mathrm{H}}>10^{24} \mathrm{~cm}^{-2}\right)$, it is impossible to estimate precisely the intrinsic X-ray luminosity (e.g. Maiolino et al. 1998; Bassani et al. 1999; Shu et al. 2006).

It has been shown that the detectability of HBLRs in Sy2s is found to be related with the IRAS $f_{60} / f_{25}$ flux ratio (Heisler, Lumsden \& Bailey 1997; Lumsden, Alexander \& Hough 2004) and the AGN activity (Sy2s with HBLRs have higher AGN luminosities, e.g. Lumsden \& Alexander 2001; Gu \& Huang 2002; Tran 2001, 2003). Some authors claimed that Sy2s without HBLRs have larger $N_{\mathrm{H}}$ (e.g. Lumsden, Alexander \& Hough 2004) and some did not (e.g. Gu \& Huang 2002; Tran 2001). Recently, Shu et al.(2006) suggested that the detectability of HBLRs for luminous Sy2s is related to $N_{\mathrm{H}}$. For a small spectropolarimetric sample (from Tran 2001), Nicastro et al.(2003) found that the detectability of HBLRs in Sy2s is regulated by the Eddington ratio. Laor (2003) also proposed an analogous model for the existence of BLRs in AGNs.

In this paper, we calculate the Eddington ratios for a larger Sy2s sample (mainly from Gu \& Huang 2002) to test the suggestion that the presence of HBLRs in Sy2s is dependent upon the Eddington ratios. In section 2, we briefly introduce the sample and present the calculation of Eddington ratios. Our results and discussion are given in Sec. 3.

\section{THE SAMPLE AND EDDINGTON RATIOS}

$\mathrm{Gu} \&$ Huang (2002) collected host-galaxy morphological types and multi-wavelength data (radio, infrared, optical, and hard X-ray) for a sample of 90 Seyfert 2 galaxies with spec-

${ }^{1}$ Key Laboratory for Particle Astrophysics, Institute of High Energy Physics, Chinese Academy of Sciences, Beijing 100039, China

2 Department of Physics and Institute of Theoretical Physics, Nanjing Normal University, Nanjing 210097, China

${ }^{3}$ Department of Astronomy, Nanjing University, Nanjing 210093, China 
tropolarimetric observations. Out of these 90 objects, 41 show HBLRs, and 49 do not (see their Tables 1 and 2). The HBLR/non-HBLR classification is mostly from the results of Tran $(2001 ; 2003)$.

In order to calculate the Eddington ratios, $L_{\mathrm{bol}} / L_{\mathrm{Edd}}$, where $L_{\mathrm{Edd}}=1.26 \times 10^{38} M_{\mathrm{BH}} / \mathrm{M}_{\odot}$ erg s${ }^{-1}$, we need to know the SMBH mass and the bolometric luminosity. As we know, recent important progress on AGN study is that we can derive more reliable SMBHs' masses through several empirical methods. The broad emission lines from BLRs (e.g. H $\beta, \mathrm{Mg}$ II, CIV, $\mathrm{H} \alpha$ ), the reverberation mapping method, the empirical BLR size-luminosity relation and the SMBHs mass-stellar velocity dispersion relation (the $M_{\mathrm{BH}}-\sigma_{*}$ relation) can be used to derive the virial SMBHs masses in type 1 AGNs (e.g. Kaspi et al. 2000, 2005; Wang \& Lu 2001; Gu et al. 2001; Vestergaard 2002; McLure \& Jarvis 2002; Bian \& Zhao 2004; Wu et al. 2004; Greene \& Ho 2006a; Nelson et al. 2001; Tremaine et al. 2002; Greene \& Ho 2006a, 2006b; Bian et al. 2006).

Because of the absence of the broad emission lines from BLRs in Sy2s, we use the $M_{\mathrm{BH}}-\sigma_{*}$ relation to derive the SMBHs mass (Tremaine et al. 2002), which is $M_{\mathrm{BH}}\left(\mathrm{M}_{\odot}\right)=$ $10^{8.13}\left[\sigma_{*} /\left(200 \mathrm{~km} \mathrm{~s}^{-1}\right)\right]^{4.02}$. The stellar velocity dispersions $\left(\sigma_{*}\right)$ are collected from the recent literature, mainly from Nelson \& Whittle (1995) and Garcia-Rissmann et al. (2005), the latter measured $\sigma_{*}$ through fitting Ca II $\lambda \lambda$ 8498, 8542, 8662 triplet. For the common objects in Nelson \& Whittle (1995) and Garcia-Rissmann et al. (2005), the values of $\sigma_{*}$ are almost the same and we adopted the new values by Garcia-Rissmann et al. (2005) for their smaller error bars. Thus, we obtained $\sigma_{*}$ for 20 HBLRs Sy2s and 25 non-HBLRs Sy2s. Because the NLRs dynamics is primarily dominated by the bulge gravitational potential (e.g. Whittle 1992; Nelson \& Whittle 1996; Greene \& Ho 2005), we also used full width at half-maximum (FWHM) of [O III] $\lambda 5007$ as a proxy for $\sigma_{*}$ for Sy2s without direct $\sigma_{*}$ measurement, $\sigma_{*}=F W H M_{[\mathrm{O} I \mathrm{II}]} / 2.35 / 1.34$, where 1.34 is a factor between the stellar velocity dispersion and the gas velocity dispersion (Greene \& Ho 2005). We obtained $\sigma_{*}$ from [O III] FWHM for 4 HBLRs Sy2s and 11 non-HBLRs Sy2s. Finally, we could calculate the SMBHs masses for 24 HBLRs and 36 non-HBLRs Sy2s.

Following Kauffmann et al. (2003), we use the [O III] luminosity as a tracer of the AGN intrinsic luminosity (Zakamsa et al. 2003a; Heckman et al., 2004; Greene \& Ho 2005). The extinction-corrected luminosity of [O III] $\lambda 5007$ is given as $L_{[\mathrm{O} \text { III] }}=4 \pi D^{2} F_{[\mathrm{OIII}]}^{\mathrm{cor}}$, where $\mathrm{F}_{[\mathrm{OIII}]}^{\mathrm{cor}}$ is the extinction-corrected flux of $[\mathrm{O}$ III $] \lambda 5007$ emission line derived from the relation (Bassani et al. 1999)

$$
F_{[\mathrm{OIII}]}^{\mathrm{cor}}=F_{[\mathrm{OIII}]}^{\mathrm{obs}}\left[\frac{\left(\mathrm{H}_{\alpha} / \mathrm{H}_{\beta}\right)_{\mathrm{obs}}}{\left(\mathrm{H}_{\alpha} / \mathrm{H}_{\beta}\right)_{0}}\right]^{2.94}
$$

where an intrinsic Balmer decrement $\left(\mathrm{H}_{\alpha} / \mathrm{H}_{\beta}\right)_{0}=3.0$ is adopted. The bolometric luminosity is calculated by assuming $L_{\text {bol }}=3500 L_{\text {[OIII] }}$ (with an uncertainty of 0.4 dex, Heckman et al., 2004). At last we calculate the Eddington ratios, $L_{\mathrm{bol}} / L_{\mathrm{Edd}}$, for this larger sample of Sy2s.

The uncertainties in the Eddington ratios depend on the uncertainties in black hole masses and bolometric luminosities. The uncertainties of SMBHs are from $\sigma_{*}$ and $M_{\mathrm{BH}}-\sigma_{*}$ relation (0.3 dex, Tremaine et al. 2002). The uncertainty of $\sigma_{*}$ is typically about $20 \mathrm{~km} \mathrm{~s}^{-1}$, which would lead to uncertainty of about 0.3 dex in the logarithm of SMBH mass. Combined with the uncertainties of $M_{\mathrm{BH}}-\sigma_{*}$ relation and the bolometric luminosity, the uncertainties of the Eddington ratio is about 0.5 index. Recently, Zhang \& Wang (2006) used the [O III] FWHM to calculated the SMBHs masses of Sy2s and suggested that absorbed non-HBLRs Sy2s harbor less massive black hole with higher accretion rates, which are similar to narrow-line Seyfert 1 galaxies (NLS1s). They found that the mean Eddington ratio of non-HBLRs Sy2s is $0.23 \pm 0.14$, which is larger than ours $(-0.47 \pm 0.16)$. The difference is due to the SMBHs masses. Their SMBHs masses were derived from the $\mathrm{FWHM}_{[\mathrm{O} \text { III }]}$ while ours are mainly from the stellar velocity dispersion. In Figure 1, we showed the relation between the [O III] FWHM and $\sigma_{*}$ for the common objects. The solid line showed $\sigma_{*}=F W H M_{[\mathrm{OIII}]} / 2.35 / 1.34$. It is obvious that, for most objects in Zhang \& Wang (2006), the [O III] FWHM underestimates $\sigma_{*}$.

In Table 1 and Table 2, we presented the multi-wavelength properties of non-HBLRs Sy2s and HBLRs Sy2s. In Table 3, we presented the distributions of SMBHs masses and the Eddington ratios for Sy2s with and without HBLRs.

\section{RESULTS AND DISCUSSION}

\subsection{Distributions of SMBHs masses and the Eddington ratios}

In Figure 2, we show the distributions of the SMBHs masses and the Eddington ratios for HBLRs Sy2s and non-HBLRs Sy2s. The distribution of the SMBHs masses is almost the same for HBLRs Sy2s and non-HBLRs Sy2s. For the whole sample, the mean Eddington ratio for HBLRs Sy2s is larger than nonHBLRs Sy2s by the magnitude of 0.40 , which is much smaller than the standard deviation of 0.79 (see Table 3 ).

In order to show the similarity between the distributions for the Eddington ratios and the SMBHs masses, we used the two sample Kolmogorov-Smirnov (K-S) test, kolmov task in IRAF 4. The K-S test showed that the distributions of the Eddington ratios/SMBHs masses for non-HBLRs Sy2s and HBLRs Sy2s subsamples are drawn from the same parent population with the probability of $48.5 \%$ and $64.8 \%$, respectively (see Table 3 for detail). Therefore, for the whole sample, there is no significant difference in the Eddington ratios/SMBHs masses between non-HBLRs Sy2s and HBLRs Sy2s.

Due to the complicated nature of unabsorbed Sy2s $\left(N_{\mathrm{H}}<\right.$ $10^{22} \mathrm{~cm}^{-2}$, Panessa \& Bassini 2002; Gallo et al. 2006 ), we also calculated the distributions of the SMBHs masses and the Eddington ratios only for HBLRs Sy2s and non-HBLRs Sy2s with $N_{\mathrm{H}}>10^{22} \mathrm{~cm}^{-2}$. The distributions of SMBHs masses and the Eddington ratios keep nearly the same as for the whole Sy2s sample.

As we showed in Section 2, the [O III] FWHM underestimated $\sigma_{*}$ if we used the formulae, $\sigma_{*}=$ $F W H M_{[\mathrm{OIII}]} / 2.35 / 1.34$. In our sample, there are 15 objects without direction $\sigma_{*}$ measurements. Excluding these $15 \mathrm{ob}-$ jects, we perform the K-S test to Sy2s with direct $\sigma_{*}$ measurements, the results are almost the same, which is presented in Table 3 as case $\mathrm{C}$.

\subsection{An Eddington ratio threshold for HBLRs and non-HBLRs Sy2s?}

As we mentioned in Section 1, AGN activity is required to understand the difference of Sy2s types. AGN luminosity is

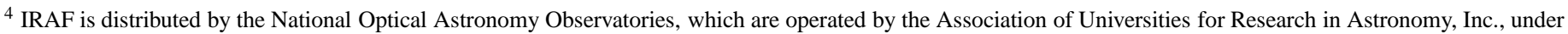
cooperative agreement with the National Science Foundation. 
mainly from the disk accretion onto central SMBHs. Some authors discussed the relation between the formation of BLRs and the disk accretion process (e.g. Nicastro 2000; Bian \& Zhao 2002). Nicastro (2000) suggested that BLRs were formed by accretion disk instabilities occurring in the critical radius at which the disk changed from gas pressure dominated to radiation pressure dominated. This radius diminishes with decreasing low enough accretion rates and BLRs can't form. Laor (2003) also proposed an analogous model that the existence of BLRs in AGNs was based on the observed upper limit of emission line width of $25,000 \mathrm{~km} \mathrm{~s}^{-1}$. Nicastro et al. (2003) used the relation between the SMBHs masses and the Hubble-typecorrected bulge luminosity to calculate the SMBHs masses. They then derived the Eddington ratio from the $2-10 \mathrm{keV} \mathrm{X}$ ray luminosity $\left(L_{\mathrm{X}}\right)$ by assuming a conversion factor, $L_{\mathrm{bol}} / L_{\mathrm{X}}$, of 10. They argued that Sy2s with HBLRs have the Eddington ratio larger than the threshold of $10^{-3}$, while non-HBLRs Sy $2 \mathrm{~s}$ lie below this threshold (see their Figure 2).

Though 2-10keV hard X-ray luminosity is a direct estimator of the AGN activity, and [O III] $\lambda 5007$ luminosity represents only an indirect (i.e., reprocessed) measurement of the nuclear activity, due to the large absorbing column density of Sy2s, it is very hard and time-consuming to derive X-ray data for a large sample of Sy2s. On the other side, Kauffmann et al. (2003) have shown that extinction-corrected $L_{[\mathrm{O} \text { III] }}$ is a good indicator of AGN activity for Type II AGNs. Here we obtained more reliable SMBHs masses from the Tremaine's $M_{\mathrm{BH}}-\sigma_{*}$ relation, and the bolometric luminosity from the extinctioncorrected [O III] $\lambda 5007$ luminosity. We used $\left.L_{\text {bol }}=3500 L_{[\mathrm{O}} \mathrm{III}\right]$ and Nicastro et al. (2003) used $L_{\text {bol }}=10 L_{X}$. However, some larger samples showed that $\left.\log \left(L_{\mathrm{X}} / L_{[\mathrm{O}} \mathrm{III}\right]\right) \sim 1$ dex $($ e.g. Heckman et al. 2005$)$, therefore the $L_{\text {bol }} / L_{\text {Edd }}$ from Nicastro et al. (2003) would be lower than ours by $\sim 1.5 \mathrm{dex}$. In Nicastro et al. (2003), the threshold of Eddington ratio is corresponding to the $L_{X} / L_{\text {Edd }}$ threshold of $10^{-4}$. In order to test the suggestion of Nicastro et al. (2003), we have to transform the $L_{X} / L_{\text {Edd }}$ threshold into the $L_{\text {bol }} / L_{\text {Edd }}$ threshold.

In the left panel of Figure 3, we showed $L_{\mathrm{X}}$ versus $\left.L_{[\mathrm{O}} \mathrm{III}\right]$. The red squares denote the HBLRs Sy2s and the black squares for the non-HBLRs Sy2s. The solid line is the best fit with the fixed slope index of $\left.1.0, \log L_{[\mathrm{O}} \mathrm{III}\right]=(-0.91 \pm 0.14)+\log L_{\mathrm{X}}$, with the correlation coefficient $(\mathrm{R})$ of 0.71 and a null probability of $P_{\text {null }}<0.001$. The dash line is the best fit with unfixed slope index, $\left.\log L_{[\mathrm{O}} \mathrm{III}\right]=(15.18 \pm 5.11)+(0.62 \pm 0.12) \log L_{\mathrm{X}}$, $\mathrm{R}=0.71$ and $P_{\text {null }}=0.001$. For two local AGN samples selected by the [O III] flux and the hard X-ray luminosity, Heckman et al. (2005) found the relation between the hard X-ray luminosity and the [O III] luminosity, they found that the mean value of $\left.\log \left(L_{\mathrm{X}} / L_{[\mathrm{O}} \mathrm{III}\right]\right)$ for Sy2s is 0.57 with a standard deviation of $1.06 \mathrm{dex}$ (see their Figure 3). Our result is different from theirs. The reason is that they didn't perform the intrinsic dust extinction correction for [O III] flux. Recently, Netzer et al. (2006) find that $L_{[\mathrm{O} \text { III }]} / L_{\mathrm{X}}$ decrease with $L_{\mathrm{X}}$, and give $\left.\log \left(L_{[\mathrm{O}} \mathrm{III}\right] / L_{\mathrm{X}}\right)=(15.0 \pm 4.0)-(0.38 \pm 0.09) \log L_{\mathrm{X}}$, which is very similar to our results (see also Shu et al. 2006).

In the right panel of Figure 3 , we show $L_{\mathrm{X}} / L_{\mathrm{Edd}}$ versus $L_{\text {bol }} / L_{\text {Edd }}$. The solid line is the best fit with the fixed slope index of $1.0, \log \left(L_{\mathrm{bol}} / L_{E d d}\right)=(2.63 \pm 0.18)+\log \left(L_{\mathrm{X}} / L_{\mathrm{Edd}}\right)$, $\mathrm{R}=0.58$ and $P_{\text {null }}<0.001$. The dash line is the best fit with unfixed slope index, $\log \left(L_{\text {bol }} / L_{\text {Edd }}\right)=(0.95 \pm 0.52)+(0.47 \pm$ $0.16) \log \left(L_{\mathrm{X}} / L_{\mathrm{Edd}}\right), \mathrm{R}=0.58$ and $P_{\text {null }}=0.006$. Considering the relation between $L_{\mathrm{X}} / L_{\mathrm{Edd}}$ and $L_{\mathrm{bol}} / L_{\mathrm{Edd}}$ (the solid line in the right panel of Figure 3), the $\log \left(L_{\mathrm{X}} / L_{\mathrm{Edd}}\right)$ threshold of -4 is corresponding to the $\log \left(L_{\mathrm{bol}} / L_{\mathrm{Edd}}\right)$ threshold of $-1.37 \pm 0.18$.

Following Nicastro et al. (2003), in Figure 4 we show the distribution of SMBHs masses (left panel) and the extinctioncorrected [O III] $\lambda 5007$ luminosity (right panel) versus the Eddington ratios. With the larger sample, there is no obvious boundary in $L_{\text {bol }} / L_{\text {Edd }}$ (the threshold of $10^{-1.37 \pm 0.18}$ ) between HBLRs Sy2s and non-HBLRs Sy2s. Below the Eddington ratio threshold of $10^{-1.37}$, there are only seven objects, all but one object (NGC513) belong to non-HBLRs Sy2s. Thus it suggested that at lower Eddington ratio, few HBLRs Sy2s are found, which is consistent with the model of Nicastro et al. (2003). However, we note that at higher Eddington ratio, distribution of SMBHs masses and Eddington ratios are nearly the same for HBLRs Sy2s and non-HBLRs Sy2s (see Figure 4).

\subsection{Other factors on the detectability of HBLRs in $S y 2 s: N_{\mathrm{H}}$ [O III]/X-ray luminosity, IRAS color $f_{60} / f_{25}$}

Because spectropolarimetry just deals with a few percent of the light from the object, detectability of HBLRs strongly depends observationally on a variety of factors, such as the dilution of the polarized signal by host galaxy light, sensitivity and the inclination of the torus to the line of sight. It happens that objects previously thought to be non-HBLR do in fact show broad polarized lines in deeper observations (e.g., Moran et al 2001). Here we just use their HBLR/non-HBLR classification from the sample of $\mathrm{Gu} \&$ Huang (2002), which is mostly from Tran (2001; 2003).

As we mentioned in the section of introduction, for Compton-thick Sy2s $\left(N_{\mathrm{H}}>10^{24} \mathrm{~cm}^{-2}\right)$, it is not possible to estimate precisely the intrinsic X-ray luminosity. In Figure 5, Compton-thick Sy2s are located above the solid horizon line of $N_{\mathrm{H}}=10^{24} \mathrm{~cm}^{-2}$ and Compton-thin Sy2s are below this line. In the left-hand panel in Figure 5 , we show $N_{\mathrm{H}}$ versus $L_{\text {bol }} / L_{\text {Edd }}$. If just considering the Compton-thin Sy2s, we find that all three objects below the threshold of $10^{-1.37}$ are non-HBLRs Sy2s. Although only a few data points, there is a trend that at smaller Eddington ratio, more non-HBLRs Sy2s can be found. Above the threshold of $10^{-1.37}$, there are 10 HBLRs Sy2s and 7 nonHBLRs Sy2s with $L_{\text {bol }} / L_{\text {Edd }}$ above the threshold of $10^{-1.37}$. Thus at larger Eddington ratio, the detectability of HBLRs Sy2s and no-HBLRs Sy2s are almost the same (see Figure 5). There are four unabsorbed (3 non-HBLRs and 1 HBLR) Sy2s below the solid horizon line of $N_{\mathrm{H}}=10^{22} \mathrm{~cm}^{-2}$ in the left-panel of Figure 5. Two unabsorbed non-HBLRs Sy2s have the Eddington ratio larger than the threshold of $10^{-1.37}$.

In the right-hand panel in Figure 5, we show $N_{\mathrm{H}}$ versus $\left.L_{[\mathrm{O}} \mathrm{III}\right]$. For Compton-thin Sy2s with lower [O III] luminosity $\left(<10^{41} \mathrm{erg} \mathrm{s}^{-1}\right)$, we find that there are only one HBLRs Sy2s and 8 non-HBLRs Sy2s, suggesting higher fraction of Compton-thin non-HBLRs Sy2s. In fact, for the lowluminosity sources $\left(<10^{41} \mathrm{erg} \mathrm{s}^{-1}\right)$, there are only two HBLRs Sy2s and 10 non-HBLRs Sy2s, nearly all of them do not show HBLRs regardless of $N_{H}$, suggesting that AGN luminosity plays a major role in the low-luminosity end. For Comptonthin Sy2s with higher [O III] luminosity $\left(>10^{41} \mathrm{erg} \mathrm{s}^{-1}\right)$, we find that there are 17 HBLRs Sy2s and 3 non-HBLRs Sy2s, suggesting higher detectability of Compton-thin HBLRs Sy2s $(\sim$ $85 \%$ ). However, for Compton-thick Sy2s with higher [O III] luminosity $\left(>10^{41} \mathrm{erg} \mathrm{s}^{-1}\right)$, we found that there are 8 HBLRs Sy $2 \mathrm{~s}$ and 10 non-HBLRs Sy2s, suggesting the detectability fraction is much smaller than Compton-thin sources. These results are 
consistent with the recent work by Shu et al. (2006). Zakamsa et al. (2003b) conducted spectropolarimetry of 12 type II (obscured) quasar candidates selected from Sloan Digital Sky Survey (SDSS). The $L_{[\mathrm{O} \text { III] }}$ luminosity of these candidates were about $10^{9} \mathrm{~L}_{\odot}, \sim 10^{42} \mathrm{erg} \mathrm{s}^{-1}$, larger than our adopted value of $10^{41} \mathrm{erg} \mathrm{s}^{-1}$. Polarizations were detected in all objects and HBLRs were safely detected in five objects.

It has been showed that HBLRs Sy2s have smaller values of $f_{60} / f_{25}$, and smaller Balmer decrements as measured by the ratio of the narrow lines, $\mathrm{H} \alpha / \mathrm{H} \beta$, compared to non-HBLRs Sy2s (Heisler, Lumsden \& Bailey 1997). The mean value of $f_{60} / f_{25}$ is $5.08 \pm 0.39$ for non-HBLRs Sy2s and $2.50 \pm 0.21$ for HBLRs Sy2s. Just considering the absorbed Sy2s $\left(N_{\mathrm{H}}>\right.$ $\left.10^{22} \mathrm{~cm}^{-2}\right)$, the difference of the $f_{60} / f_{25}$ distribution is more significant ( $5.62 \pm 0.67$ for non-HBLRs Sy2s and $2.46 \pm 0.23$ for HBLRs Sy2s). And K-S tests show that the $f_{60} / f_{25}$ distributions for non-HBLRs Sy2s and HBLRs Sy2s subsamples are drawn from the same parent population with a possibility of 0.0037 and 0.0031 for the whole sample and for objects with $N_{\mathrm{H}}>10^{22} \mathrm{~cm}^{-2}$, respectively. A plot of $L_{\text {bol }} / L_{\text {Edd }}$ vs. $f_{60} / f_{25}$ showed that the correlation would be nice for Compton-thin Sy2s $\left(\mathrm{R}=-0.37, P_{\text {null }}=0.03\right)$. The solid line is the best fit, $f_{60} / f_{25}=(4.22 \pm 0.54)-(1.18 \pm 0.52) \log \left(L_{\text {bol }} / L_{\text {Edd }}\right)$ (see Figure 6). However, this correlation becomes weaker $(\mathrm{R}=-0.32$, $\left.P_{\text {null }}=0.02\right)$ for the whole Sy2s sample. Near/mid infrared emission is regarded as being anisotropic whereas far infrared is isotropic. $f_{60} / f_{25}$ can be used as an orientation indicator of torus (Heisler et al. 1997). The larger values of $f_{60} / f_{25}$ showed the higher tours inclination to the line of sight. The median correlation between $f_{60} / f_{25}$ and $L_{\text {bol }} / L_{\text {Edd }}$ showed that the larger Eddington ratio would lead to smaller torus opening angle (e.g. Wang et al. 2005), and cooler infrared color is expected (smaller $f_{60} / f_{25}$ ).

\section{CONCLUSION}

We calculated the SMBHs masses and the Eddington ratios for a larger compiled Sy2s sample. For Sy2s with the Eddington ratio larger than the threshold of $10^{-1.37}$, there is no obvious discrimination in the Eddington ratios/black hole masses for Sy2s with and without HBLRs; Sy2s with low luminosity and low Eddington ratios do not show HBLRs regardless of $N_{\mathrm{H}}$, which is consistent with the prediction of Nicastro et al. (2003). For high-luminosity Compton-thin Sy2s, we find very higher detectability of HBLRs Sy2s $(\sim 85 \%)$. However, as the present sample in this paper is an amalgamation of different observations with diverse quality of spectropolarimetric data, varying from object to object determined by the brightness, observers, integration time, and a host of other factors, it is hard to entangle the physical nature of Sy2s with and without HBLRs. In the future, we need more new data of hard X-ray spectra with good quality optical spectropolarimetric information.

\section{ACKNOWLEDGMENTS}

We thank the anonymous referee for her/his suggestions. This work has been supported by the NSFC (Nos. 10403005, 10473005, 10325313, 10233030 and 10521001) and the Science-Technology Key Foundation from Education Department of P. R. China (No. 206053). QGU would like to acknowledge the financial supports from China Scholarship Council (CSC) and the National Natural Science Foundation of China under grants 10103001, 10221001 and 10633040 . We are grateful to Dr. Jian-Min Wang for very helpful suggestions.

\section{REFERENCES}

Antonucci, R., 1993, ARA\&A, 31, 473

Antonucci, R., Miller J.S., 1985, ApJ, 297, 621

Baskin, A., Laro, A., 2005, MNRAS, 358, 1043

Bassani, L., et al. 1999, ApJS, 121, 473

Bian, W., Gu, Q., Zhao, Y., et al., 2006, MNRAS, 372, 876

Bian, W., Zhao, Y., 2002, A\&A, 395, 465

Bian, W., Zhao, Y., 2004, MNRAS, 347, 607

Gallo, L. C., et al., 2006, MNRAS, 365, 688

Garcia-Rissmann, A., Vega, L. R., Asari, N. V., et al., 2005, MNRAS, 359, 765

Gelderman, R., Whittle M., 1994, ApJS, 91, 491

Greene, J. E., Ho, L. C., 2005, ApJ, 627, 721

Greene, J. E., Ho, L. C., 2006a, ApJL, 641, L21

Greene, J. E., Ho, L. C., 2006b, ApJ, 641, 117

Gu, M, Cao, X., Jiang D., 2001, MNRAS, 327, 111

Gu, Q., Huang, J., 2002, ApJ, 579, 205

Heckman, T. M., Kauffmann, G., Brinchmann, J., Charlot S., Tremonti C., White, S. D. M., 2004, ApJ, 613, 109

Heckman, T. M., Ptak, A., Hornschemeier, A., Kauffmann, G., 2005, ApJ, 634, 161

Heisler, C. A., Lumsden, S. L., Bailey, J. A. 1997, Nature, 385, 700

Kaspi, S., Maoz, D., Netzer, H., Peterson, B.M., Vestergaard, M., \& Jannuzi, B.T. 2005, ApJ, 629, 61

Kaspi, S., Smith, P.S., Netzer, H., Maoz, D., Jannuzi, B.T., Giveon, U., 2000, ApJ, 533, 631

Laor, A. 2003, ApJ, 590, 86

Lipari, S., Bonatto, C., Pastoriza, M.G., 1991, MNRAS, 253, 19

Lumsden, S.L., Heisler, C.A., Bailey, J.A., Hough, J.H., Young, S., 2001, MNRAS, 327,459

Lumsden, S., Alexander, D., Hough, J., 2004, MNRAS, 348, 1451

Maiolino, R., et al. 1998, A\&A, 338, 781
McElroy, D. B., 1995, ApJS, 100, 105

McLure, R. J., Jarvis, M. J., 2002, MNRAS, 337, 109

Moran, E.C., Barth, A.J., Kay, L.E., Filippenko, A.V., 2000, ApJ, 540, L73

Moran, E. C., Kay, L. E., Davis, M., Filippenko, A. V., \& Barth, A. 2001, ApJ, 556, L75

Nelson, C.H., Whittle, M., 1995, ApJS, 99, 67

Nelson, C. H., 2001, ApJ, 544, L91

Netzer, H. et al. 2006, A\& A, in press, astro-ph/0603712

Nicastro, F., 2000, ApJ, 530, L65

Nicastro, F., Martocchia A., Matt G., 2003, ApJ, 589, L13

Panessa, F. \& Bassani, L., 2002, A\&A, 394, 435

Rees, M. J. 1984, ARA\&A, 22, 471

Shu, et al., 2006, ApJ, in press, astro-ph/0603338

Tran, H. D., 1995, ApJ, 440, 565

Tran, H. D., 2001, ApJ, 554, L19

Tran, H. D., 2003, ApJ, 583, 632

Tremaine, S., et al., 2002, Ap J, 574, 740

Urry, C. M., Padovani, P., 1995, PASP, 107, 803

Veilleux, S., Goodrich, R. W., Hill, G. J., 1997, ApJ, 477, 631

Vestergaard, M., 2002, ApJ, 571, 733

Wang, J. M., Zhang E. P., Luo B., 2005, ApJ, 627, L5

Wang, T. G., Lu, Y. J., 2001, A\&A, 377, 52

Whittle, M. 1992, ApJS, 79, 49

Wilson, A.S., Nath, B., 1990, ApJS, 74, 731

Wilson, A.S., Baldwin, J.A., 1989, AJ, 98, 205

Wu, X. B., et al., 2004, A\& A, 424, 793

Young, S., et al., 1996, MNRAS, 281, 1206

Zakamsa, N. L., et al., 2003a, AJ, 126, 2125

Zakamsa, N. L., et al., 2003b, AJ, 129, 1212

Zhang, E. P., Wang, J. M., 2006, ApJ, in press, astro-ph/0606103 
TABLE 1

THE NON-HBLRS SY2S SAMPLE.

\begin{tabular}{|c|c|c|c|c|c|c|c|c|c|}
\hline $\begin{array}{l}\begin{array}{l}\text { name } \\
\text { (1) }\end{array} \\
\text { (1) }\end{array}$ & $\begin{array}{l}\left.L_{[\mathrm{O}} \mathrm{III}\right] \\
(2)\end{array}$ & $\begin{array}{l}\sigma \\
\text { (3) }\end{array}$ & $\begin{array}{l}\text { Ref. } \\
\text { (4) }\end{array}$ & $\begin{array}{l}\log _{\mathrm{bh}} \\
(5)\end{array}$ & $\begin{array}{l}\log _{\left(L_{\mathrm{bol}} / L_{\mathrm{Edd}}\right)} \\
(6)\end{array}$ & $\begin{array}{l}f_{60} / f_{25} \\
\text { (7) }\end{array}$ & $\begin{array}{l}\log N_{H} \\
(8)\end{array}$ & $\begin{array}{l}\log _{\mathrm{X}}{ }_{\mathrm{X}} \\
\text { (9) }\end{array}$ & $\begin{array}{l}{\log \left(L_{X} / L_{\text {Edd }}\right)}_{(10)} \\
(10)\end{array}$ \\
\hline Mrk334 & 41.289 & 79 & 4 & 6.51 & 0.22 & 4.04 & 20.64 & 43.10 & -1.51 \\
\hline NGC34 & 42.772 & 105 & 3 & 7.00 & 1.22 & 6.76 & & - & - \\
\hline IRAS00198-7926 & 42.562 & 239 & 5 & 8.44 & -0.43 & 2.49 & $>24$ & - & - \\
\hline Mrk573 & 42.001 & $123 *$ & 1 & 7.28 & 0.16 & 1.59 & & - & - \\
\hline NGC1144 & 42.251 & $219 *$ & 2 & 8.29 & $\begin{array}{l}-0.59 \\
-0.10\end{array}$ & 8.72 & 20.70 & $<43.28$ & -3.11 \\
\hline Mrk1066 & 42.175 & $105 *$ & 1 & 7.01 & 0.61 & 4.86 & $>24$ & - & - \\
\hline NGC1241 & 42.472 & $136 *$ & 2 & 7.46 & 0.46 & 8.29 & - & - & - \\
\hline NGC1320 & 40.959 & $116 *$ & 1 & 7.18 & -0.78 & 2.19 & - & - & - \\
\hline NGC1358 & 40.783 & $173 *$ & 1 & 7.88 & -1.65 & 3.05 & - & - & - \\
\hline NGC1386 & 40.586 & $120 *$ & 1 & 7.24 & -1.21 & 4.03 & $>25$ & - & - \\
\hline IRAS03362-1642 & 41.529 & - & - & - & - & 2.13 & -2 & - & - \\
\hline IRAS04103-2838 & - & - & - & - & - & 3.39 & - & - & - \\
\hline IRAS04210+0400 & 42.328 & 127 & 6 & 7.34 & 0.44 & 2.38 & - & _- & - \\
\hline IRAS04229-2528 & 41.903 & - & 0 & - & - & 3.69 & - & - & - \\
\hline IRAS04259-0440 & 40.44 & - & - & - & - & 2.80 & - & - & - \\
\hline NGC1667 & 41.921 & $173 *$ & 1 & 7.88 & -0.51 & 8.91 & $>24$ & - & - \\
\hline NGC1685 & 42.582 & - & - & $-\infty$ & - & 4.48 & -24 & - & - \\
\hline ESO428-G014 & 41.937 & 127 & 7 & 7.34 & 0.05 & 2.49 & $>25$ & - & - \\
\hline IRAS08277-0242 & & - & & - & - & 3.45 & & - & - \\
\hline NGC3079 & 40.427 & $150 *$ & 8 & 7.63 & -1.76 & 14.01 & 22.204 & 40.25 & -5.48 \\
\hline NGC3281 & 40.998 & 123 & 3 & 7.28 & -0.84 & 2.65 & 23.903 & 42.80 & -2.58 \\
\hline IRAS $10340+0609$ & & - & & - & - & 1.56 & - & - & - \\
\hline NGC3362 & 41.269 & $92 *$ & 1 & 6.77 & -0.06 & 1.00 & - & - & - \\
\hline UGC6100 & 42.18 & $156 *$ & 1 & 7.70 & -0.07 & 2.84 & - & - & - \\
\hline NGC3660 & 40.914 & - & & - & - & 7.78 & 20.255 & 41.8 & - \\
\hline NGC3982 & 40.019 & $62 *$ & 1 & 6.09 & -0.62 & 8.26 & - & - & - \\
\hline NGC4117 & & $95 *$ & 1 & 6.83 & - & & - & - & - \\
\hline NGC4501 & 39.804 & $171 *$ & 8 & 7.86 & -2.61 & 5.95 & - & - & - \\
\hline NGC4941 & 40.894 & 80 & 3 & 6.53 & -0.19 & 4.10 & 23.653 & 40.82 & -3.81 \\
\hline NGC5135 & 42.311 & $128 *$ & 2 & 7.35 & 0.40 & 6.73 & $>24$ & & \\
\hline NGC5194 & 40.168 & $102 *$ & 1 & 6.95 & -1.34 & 6.59 & 23.699 & 39.96 & -5.10 \\
\hline NGC5256 & 41.825 & 100 & 3 & 6.92 & 0.35 & 6.36 & $>24$ & - & - \\
\hline NGC5283 & 40.836 & $148 *$ & 1 & 7.60 & -1.32 & & - & - & _- \\
\hline Mrk1361 & 42.242 & - & 1 & ${ }_{-}-0$ & - & 3.91 & - & - & - \\
\hline IRAS13452-4155 & 42.098 & 80 & 9 & 6.51 & 1.03 & 2.27 & - & - & - \\
\hline NGC5643 & 41.225 & 77 & 3 & 6.44 & 0.23 & 5.03 & $>25$ & $\overline{-}$ & - \\
\hline NGC5695 & 40.501 & $144 *$ & 1 & 7.56 & -1.61 & 4.39 & - & - & - \\
\hline NGC5728 & 42.083 & $209 *$ & 8 & 8.21 & -0.68 & 10.19 & - & - & - \\
\hline IRAS19254-7245 & 42.731 & - & - & - & - & 3.96 & $>24$ & - & - \\
\hline NGC6890 & 40.808 & 78 & 3 & 6.48 & -0.23 & 4.92 & & - & - \\
\hline IRAS20210+1121 & 43.124 & - & - & - & - & 2.42 & $>25$ & - & - \\
\hline NGC7130 & 42.477 & $141 *$ & 2 & 7.52 & 0.40 & 7.76 & $>24$ & _- & _- \\
\hline NGC7172 & 39.767 & $154 *$ & 2 & 7.67 & -2.46 & 6.37 & 22.934 & 42.50 & -3.28 \\
\hline NGC7496 & 40.211 & $76 *$ & 2 & 6.44 & -0.79 & 5.54 & 22.699 & 41.65 & -2.89 \\
\hline IRAS23128-5919 & & - & - & - & - & 6.79 & - & - & - \\
\hline IC5298 & 42.078 & - & - & - & - & 4.65 & - & - & - \\
\hline NGC7582 & 41.357 & $121 *$ & 2 & 7.25 & -0.45 & 6.90 & 23.079 & 42.14 & -3.21 \\
\hline NGC7590 & 39.949 & $93 *$ & 2 & 6.79 & -1.40 & 9.07 & 20.964 & 40.80 & -4.10 \\
\hline NGC7672 & & $98 *$ & 1 & 6.88 & - & 2.96 & & - & - \\
\hline
\end{tabular}

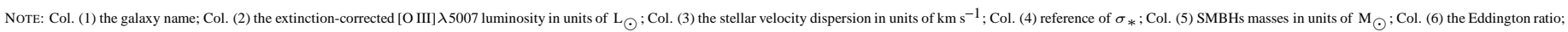

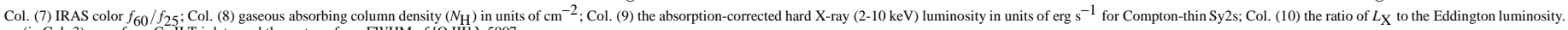
* (in Col. 3): $\sigma_{*}$ from Ca II Triplets, and the rest are from FWHM of [O III] $\lambda$ 5007.

REFERENCES.: (1) Nelson \& Whittle 1995; (2) Garcia-Rissmann 2005; (3) Whittle 1992; (4) Wilson \& Nath 1990; (5) Lipariet et al. 1991; (6) Gelderman \& Whittle 1994; (7) Wilson \& Baldwin 1989; (8) McElroy 1995; (9) Young et al. 1996.

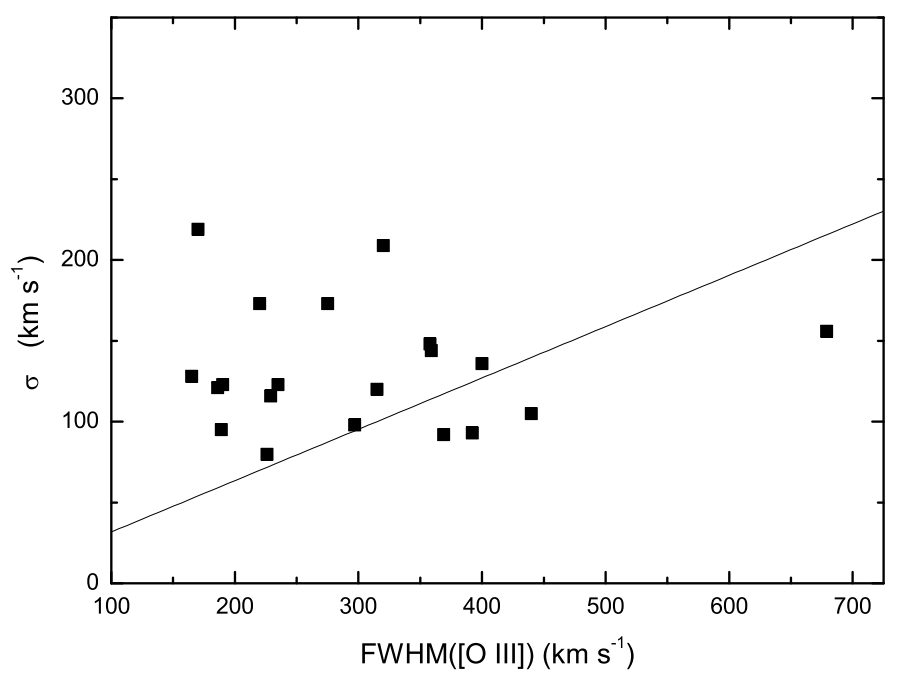

FIG. 1.- The [O III] FWHM versus $\sigma_{*}$ for the common objects in our sample and the sample of Zhang \& Wang (2006). The solid line showed $\sigma_{*}=$ $F W H M_{[\mathrm{OIII}]} / 2.35 / 1.34$. 
TABLE 2

THE HBLRS SY2S SAMPLE.

\begin{tabular}{|c|c|c|c|c|c|c|c|c|c|}
\hline $\begin{array}{l}\text { name } \\
\text { (1) }\end{array}$ & $\begin{array}{l}\left.L_{[\mathrm{O}} \mathrm{III}\right] \\
(2)\end{array}$ & $\begin{array}{l}\sigma \\
(3)\end{array}$ & $\begin{array}{l}\text { Ref. } \\
\text { (4) }\end{array}$ & $\begin{array}{l}\log M_{\text {bh }} \\
(5)\end{array}$ & $\begin{array}{l}\log _{\left(L_{\mathrm{bol}} / L_{\mathrm{Edd}}\right)} \\
(6)\end{array}$ & $\begin{array}{l}f_{60} / f_{25} \\
\text { (7) }\end{array}$ & $\begin{array}{l}\log _{\mathrm{H}} \\
(8)\end{array}$ & $\begin{array}{l}\log L_{X} \\
\text { (9) }\end{array}$ & $\begin{array}{l}\log \left(L_{X} / L_{\text {Edd }}\right) \\
(10)\end{array}$ \\
\hline Mrk348 & 41.912 & $118 *$ & 1 & 7.21 & 0.15 & 1.42 & 23.04 & 43.01 & -2.30 \\
\hline IRAS00521-7054 & 42.743 & - & - & - & - & 1.27 & & - & - \\
\hline NGC424 & 41.462 & 163 & 3 & 7.78 & -0.87 & 1.18 & 24.18 & _- & - \\
\hline NGC513 & 40.597 & $152 *$ & 1 & 7.65 & -1.61 & 6.33 & & - & - \\
\hline NGC591 & 41.954 & $95 *$ & 1 & 6.83 & 0.57 & 4.33 & : & $\overline{-}$ & $\overline{-}$ \\
\hline IRAS01475-0740 & 41.689 & - & $=$ & - & - & 1.10 & - & - & - \\
\hline NGC788 & 40.99 & $140 *$ & 1 & 7.51 & -1.07 & 0.99 & - & - & - \\
\hline NGC1068 & 42.645 & $128 *$ & 1 & 7.35 & 0.74 & 2.16 & $>25$ & - & - \\
\hline IRAS02581-1136 & 41.536 & - & - & - & - & 1.07 & - & - & - \\
\hline IRAS04385-0828 & 40.117 & - & - & - & - & 1.77 & & & - \\
\hline IRAS05189-2524 & 42.459 & - & - & - & - & 3.99 & 22.69 & 43.33 & - \\
\hline Mrk3 & 43.221 & $269 *$ & 1 & 8.65 & 0.02 & 1.30 & 24.04 & - & - \\
\hline NGC2273 & 41.449 & $124 *$ & 1 & 7.30 & -0.40 & 4.88 & $>25$ & - & - \\
\hline Mrk 1210 & $\begin{array}{r}1.1449 \\
42.195\end{array}$ & $77 *$ & 2 & 6.46 & $\begin{array}{l}-0.40 \\
1.18\end{array}$ & $\begin{array}{l}4.08 \\
0.98\end{array}$ & $>24$ & - & - \\
\hline $\begin{array}{l}\text { MGri2120 } \\
\text { NGC3081 }\end{array}$ & $\begin{array}{l}4.195 \\
41.331\end{array}$ & $129 *$ & $\frac{2}{2}$ & $\begin{array}{l}6.46 \\
7.36\end{array}$ & $\begin{array}{l}1.18 \\
-0.59\end{array}$ & 0.98 & $\begin{array}{l}>24 \\
23.82\end{array}$ & $\overline{4} 1.83$ & -3.63 \\
\hline IRAS1 1058-1131 & 42.316 & - & $=$ & - & - & 2.39 & $>24$ & - & - \\
\hline $\begin{array}{l}\text { Was } 49 \mathrm{~b} \\
\text {. }\end{array}$ & 42.412 & - & - & - & - & 1.45 & 22.799 & 42.97 & - \\
\hline $\begin{array}{l}\text { NGC4388 } \\
\text { NGC }\end{array}$ & $\begin{array}{l}4.2112 \\
41.684\end{array}$ & $119 *$ & 1 & $\overline{7} .22$ & -0.10 & $\begin{array}{l}2.45 \\
2.94\end{array}$ & 23.623 & 42.74 & -2.58 \\
\hline NGC4507 & 41.569 & $146 *$ & 2 & 7.58 & -0.57 & 3.95 & 23.462 & 43.22 & -2.46 \\
\hline IC3639 & 42.113 & $95 *$ & 2 & 6.83 & 0.73 & 2.95 & $>25$ & - & - \\
\hline MCG-3-34-64 & 42.322 & - & & - & - & 2.06 & 23.881 & 42.53 & - \\
\hline NGC5252 & 41.963 & $190 *$ & 1 & 8.04 & -0.63 & & 22.633 & 43.06 & -3.08 \\
\hline NGC5347 & 40.446 & $93 *$ & 1 & $\begin{array}{l}0.07 \\
6.79\end{array}$ & -0.90 & 1.25 & $>24$ & & \\
\hline $\begin{array}{l}\text { Nucs4i } \\
\text { Mrk463E }\end{array}$ & $\begin{array}{l}40.4460 \\
42.785\end{array}$ & 173 & 3 & $\begin{array}{l}0.19 \\
7.88\end{array}$ & $\begin{array}{l}-0.90 \\
0.35\end{array}$ & $\begin{array}{l}1.25 \\
1.51\end{array}$ & 23.204 & 42.65 & -3.33 \\
\hline $\begin{array}{l}\text { Circinus } \\
\text { nats }\end{array}$ & 40.474 & $75 *$ & 1 & 6.42 & -0.50 & 3.64 & $>24$ & - & - \\
\hline $\begin{array}{l}\text { NGC5506 } \\
\text { nat }\end{array}$ & 41.608 & 86 & 4 & 6.65 & 0.40 & 2.01 & 22.531 & 42.86 & -1.89 \\
\hline Mrk477 & 43.543 & 117 & 3 & 7.20 & 1.79 & 2.51 & $>24$ & - & - \\
\hline ESO273-IG04 & 42.372 & - & - & - & - & 2.77 & & _- & - \\
\hline NGC5929 & 40.926 & $121 *$ & 1 & 7.25 & -0.88 & 5.64 & 20.763 & 42.09 & -3.27 \\
\hline NGC5995 & 42.904 & - & - & - & -0.00 & 2.82 & 21.934 & 43.43 & -3.27 \\
\hline IRAS15480-0344 & $\begin{array}{l}2.2 .904 \\
42.946\end{array}$ & - & - & 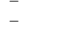 & $\overline{-}$ & 1.47 & -21.904 & -5.43 & $\overline{-}$ \\
\hline 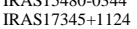 & $\begin{array}{r}42.9406 \\
42.956\end{array}$ & - & - & $\overline{-}$ & - & $\begin{array}{l}1.47 \\
2.47\end{array}$ & : & $\begin{array}{l}- \\
-\end{array}$ & $\overline{-}$ \\
\hline NGC6552 & 42.122 & - & - & - & - & 3.64 & 23.778 & 42.46 & - \\
\hline IRAS $20460+1925$ & 42.838 & - & - & - & - & 1.67 & 22.398 & 44.08 & - \\
\hline IC5063 & 41.919 & $160 *$ & 1 & 7.74 & -0.38 & 1.53 & 23.38 & 42.85 & -2.99 \\
\hline IRAS22017+0319 & 42.497 & - & - & - & - & 1.61 & 21.301 & 43.41 & \\
\hline NGC7212 & 42.636 & $137 *$ & 1 & 7.47 & 0.61 & 4.08 & 23.653 & 42.36 & -3.21 \\
\hline MCG-3-58-7 & 43.916 & - & - & - & - & 2.77 & & - & - \\
\hline IRAS $23060+0505$ & 41.692 & - & - & - & - & 2.70 & 22.924 & 44.16 & _- \\
\hline NGC7674 & 42.495 & $144 *$ & 1 & 7.56 & 0.38 & 2.81 & $>25$ & -.10 & - \\
\hline NGC7682 & 41718 & $123 *$ & 1 & 728 & -0.12 & 203 & & _ & - \\
\hline
\end{tabular}

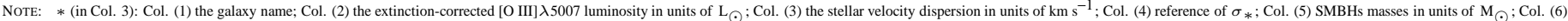

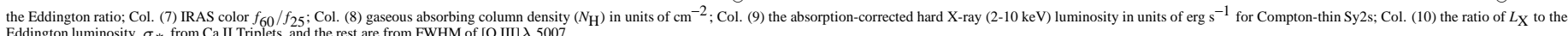

References.: (1) Nelson \& Whittle 1995; (2) Garcia-Rissmann 2005; (3) Whittle 1992; (4) Wilson \& Nath 1990; (5) Lipariet et al. 1991; (6) Gelderman \& Whittle 1994; (7) Wilson \& Baldwin 1989; (8) McElroy 1995; (9) Young et al. 1996.

TABLE 3

THE DISTRIBUTIONS OF SMBHS MASSES AND THE EDDINGTON RATIOS FOR HBLRS SY2S AND NON-HBLRS SY2S.

\begin{tabular}{lllllllll}
\hline \hline Type & $\log \left(M_{B H} / \mathrm{M}_{\odot}\right)$ & $\begin{array}{l}S D_{1} \\
(1)\end{array}$ & $\begin{array}{l}\mathrm{N} 1 \\
(2)\end{array}$ & $\begin{array}{l}\mathrm{P} 1 \\
(3)\end{array}$ & $\begin{array}{l}\log \left(L_{b o l} / L_{E d d}\right) \\
(6)\end{array}$ & $\begin{array}{l}S D_{2} \\
(7)\end{array}$ & $\begin{array}{l}\mathrm{N} 2 \\
(8)\end{array}$ & $\begin{array}{l}\mathrm{P} 2 \\
(9)\end{array}$ \\
\hline non-HBLRs Sy2s & $7.22 \pm 0.10$ & 0.57 & 36 & $64.76 \%$ & $-0.47 \pm 0.16$ & 0.93 & 34 & $48.50 \%$ \\
HBLRs Sy2s & $7.33 \pm 0.10$ & 0.51 & 24 & & $-0.07 \pm 0.16$ & 0.79 & 24 & \\
A:non-HBLRs Sy2s & $7.35 \pm 0.19$ & 0.57 & 9 & $82.41 \%$ & $-0.01 \pm 0.20$ & 0.59 & 9 & $43.46 \%$ \\
A:HBLRs Sy2s & $7.23 \pm 0.21$ & 0.67 & 10 & & $0.22 \pm 0.29$ & 0.90 & 10 & \\
B:non-HBLRs Sy2s & $7.13 \pm 0.19$ & 0.60 & 10 & $40.05 \%$ & $-0.96 \pm 0.25$ & 0.79 & 10 & $16.70 \%$ \\
B:HBLRs Sy2s & $7.44 \pm 0.13$ & 0.40 & 10 & & $-0.16 \pm 0.16$ & 0.52 & 10 & \\
C:non-HBLRs Sy2s & $7.33 \pm 0.11$ & 0.53 & 25 & $93.86 \%$ & $-0.78 \pm 0.19$ & 0.91 & 23 & $16.53 \%$ \\
C:HBLRs Sy2s & $7.33 \pm 0.12$ & 0.52 & 20 & & $-0.17 \pm 0.16$ & 0.72 & 20 & \\
\hline \hline
\end{tabular}

Note:Col.1: type; Col.2: $\log$ of SMBHs masses in units of $\mathrm{M}_{\odot}$; Col.3: the standard deviation of log of SMBHs masses; Col.4: Number; Col.5: possibility from the same parent population of the SMBHs mass distribution; Col.6: log of the Eddington ratio; Col.7: the standard deviation of $\log$ of the Eddington ratio; Col.8: Number. Col. 9: possibility from the same parent population of the Eddington ratio. A: objects with $N_{\mathrm{H}} \geq 10^{24} \mathrm{~cm}^{-2}$ and the available Eddington ratios, B: objects with $N_{\mathrm{H}}<10^{24} \mathrm{~cm}^{-2}$ and the available Eddington ratios, C: objects with the direct $\sigma_{*}$ measurements. 

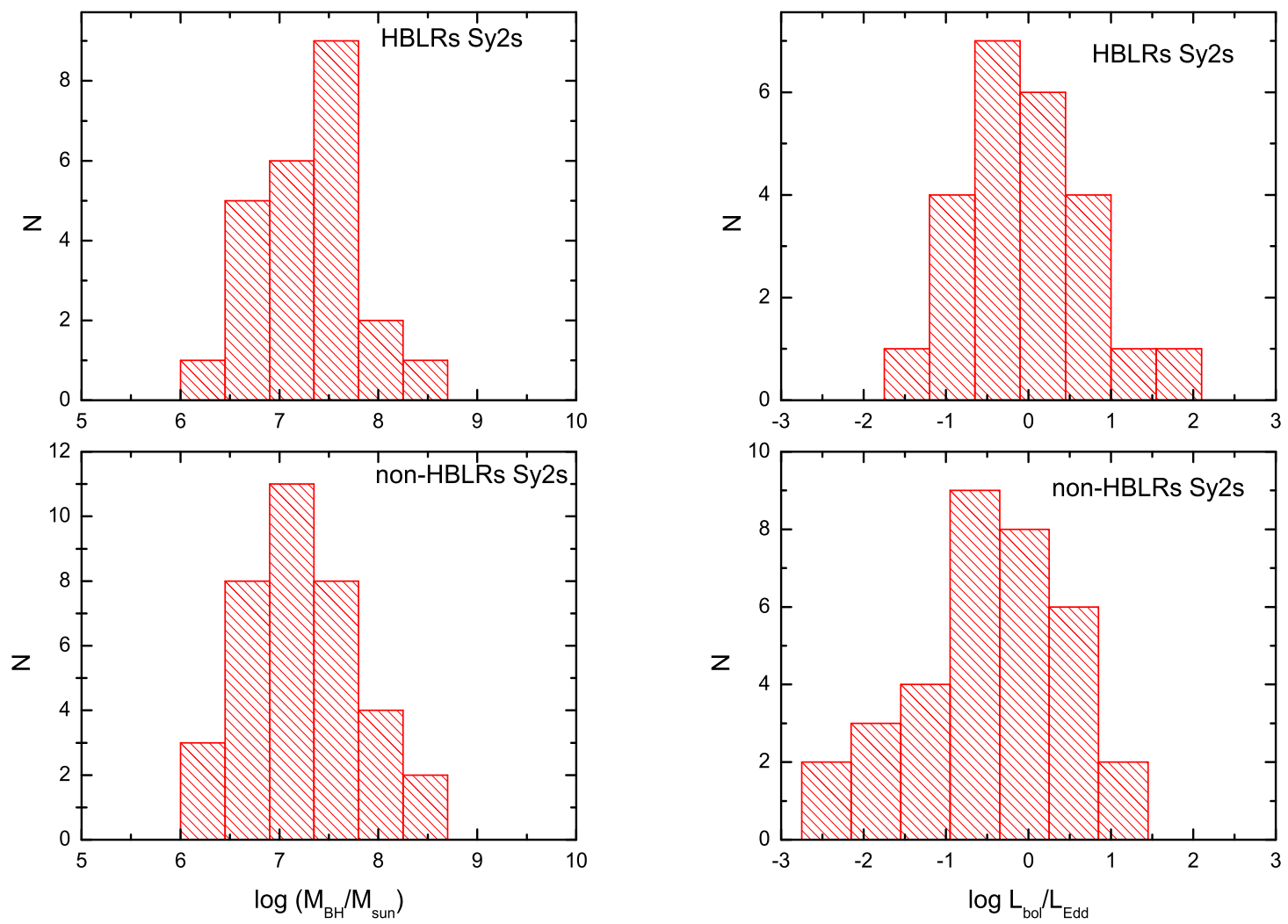

FIG. 2.- Left-hand side: the distribution of SMBHs masses for HBLRs Sy2s and non-HBLRs Sy2s. Right-hand side: the distribution of the Eddington ratios for HBLRs Sy2s and non-HBLRs Sy2s.
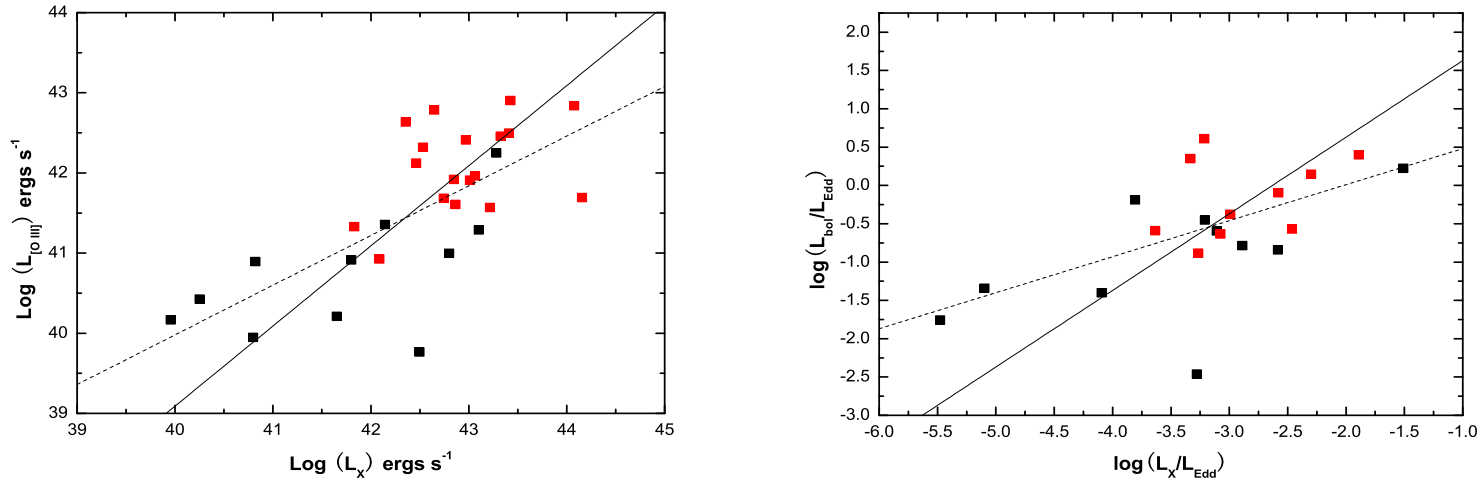

FIG. 3.- Left-hand side: $L_{\mathrm{X}}$ versus $\left.L_{[\mathrm{O}} \mathrm{III}\right]$; Right-hand side: $L_{\mathrm{X}} / L_{\mathrm{Edd}}$ versus $L_{\mathrm{bol}} / L_{\mathrm{Edd}}$. The solid line is the best fit with the fixed slope index of one and the dash line is the best fit with unfixed slope index. The red squares denote HBLRs Sy2s and the black squares denote non-HBLRs Sy2s. 

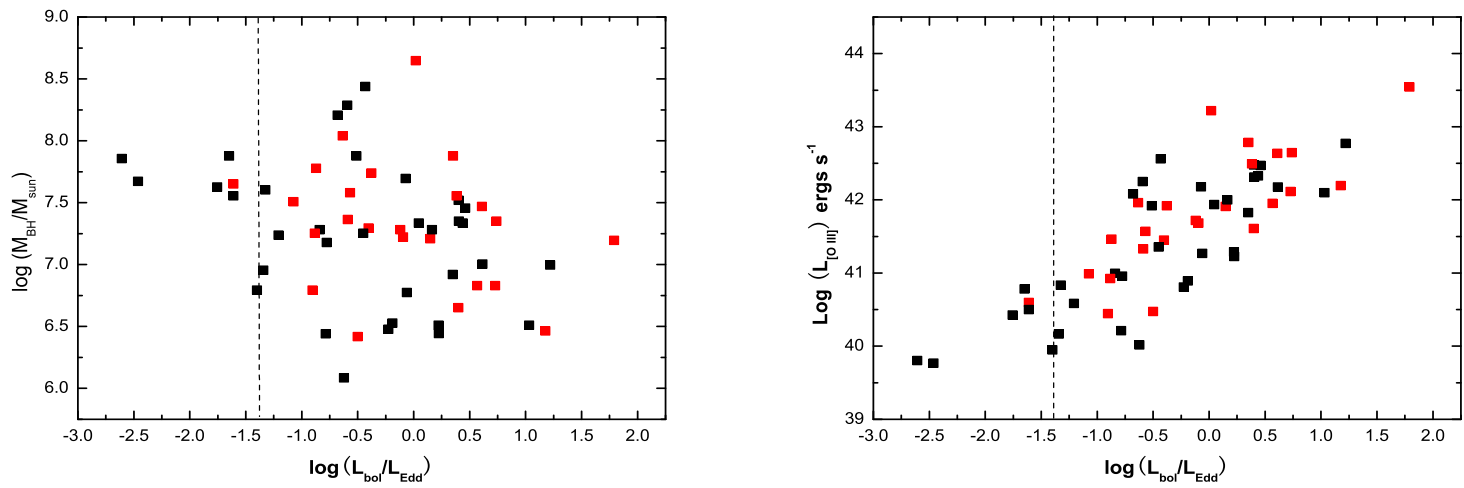

FIG. 4. - Left-hand side: the central SMBHs masses versus the Eddington ratios. Right-hand side: the [O III] $\lambda 5007$ luminosity versus the Eddington ratios. The dash line showed the $\log \left(L_{\mathrm{bol}} / L_{\mathrm{Edd}}\right)$ threshold of -1.37 . The symbols are same to that in Fig. 3.
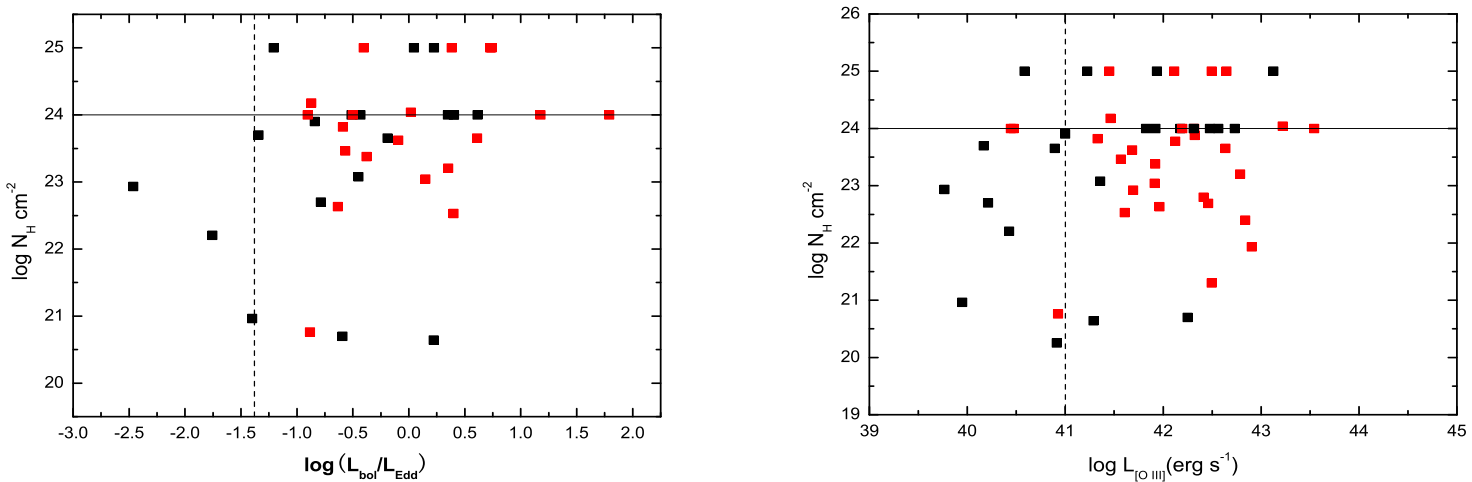

FIG. 5. - Left-hand side: $N_{\mathrm{H}}$ versus the Eddington ratios. Right-hand side: $N_{\mathrm{H}}$ versus the [O III] $\lambda 5007$ luminosity. The dash line showed the $\log \left(L_{b o l} / L_{\mathrm{Edd}}\right)$ threshold of -1.37 (left panel) and the $\left.L_{[\mathrm{O}} \mathrm{III}\right]$ threshold of $10^{41} \mathrm{ergs}^{-1}$ (right panel). The horizon solid line is the line of $N_{\mathrm{H}}=10^{24} \mathrm{~cm}^{-2}$. The symbols are same to that in Fig. 3.

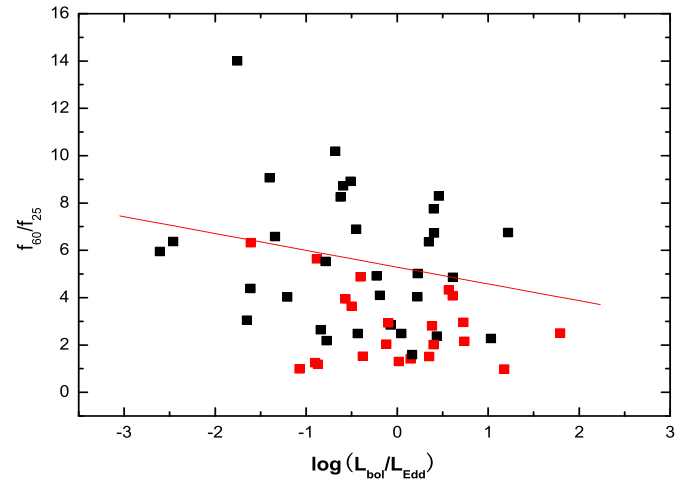

FIG. 6.- $f_{60} / f_{25}$ versus $L_{\mathrm{bol}} / L_{\mathrm{Edd}}$ for Compton-thin Sy2s $\left(N_{\mathrm{H}}<10^{24} \mathrm{~cm}^{-2}\right)$. The symbols are same to that in Fig. 3 . 